\title{
ORLANDO RIBEIRO, MAESTRO DE GEÓGRAFOS
}

POR

\author{
ÁNGEL CABO ALONSO
}

El 17 de noviembre de 1997 ha fallecido el profesor Orlando Ribeiro. Con anterioridad envié una reseña de sus Opúsculos Geográficos a la revista Ería que ya habrá visto la luz cuando el presente escrito se publique. Sin embargo, me parece que quien ha sido creador y maestro de la Geografía portuguesa requiere un comentario que comprenda algo más sobre su personalidad, su abundante bibliografía y su esforzada labor en pro de la mejora de la docencia y la investigación. Y es lo menos que merece quien tanto afecto y estima tuvo a España y a quienes nos ocupamos, de manera mucho más modesta, en similares tareas, sentimientos que en su última lección académica, en febrero de 1981, le hacían proclamar; «Hoje a Ciência espanhola vai à cabeça em muitos ramos, tanto nas Ciências humanas como nas físico-naturais, e a sua jovem Geografia adiantouse à nossa - consequência da dimensão física e dos largos recursos humanos e materiais do País irmão.» En la misma reseña de aquellos trabajos suyos menores ya me referí a esos sentimientos: huelga la reiteración. Destacaré aquí otros aspectos de su calidad humana y científica.

\section{Él y su circunstancia}

Nacido al iniciarse la segunda década del siglo, casi toda su vida se ha desarrollado dentro del curso de acontecimientos que habían sacudido e iban a sacudir con violencia a Portugal. Algo antes, en 1908,

Estudios Geográficos

Tomo LIX, n. ${ }^{\circ} 232$, julio-septiembre 
fueron asesinados en Terreiro do Paço el monarca Carlos I y el heredero. De la inseguridad que siguió al magnicidio puede dar idea el hecho de que en los dos años que duró el reinado del sucesor, don Manuel, hubo siete distintos gobiernos. Aunque la República se proclamó —en octubre de 1910 - de manera tan pacífica que los coetánesos aseveraban que se extendió de inmediato a todo el territorio mediante el telégrafo, no cesaron a continuación las revueltas. El país se vio después involucrado en la guerra europea de 1914-1918, y el general Carmona impuso en 1927 el régimen dictatorial, del que Oliveira Salazar, como jefe de gobierno, iba a ser sustentador. Advino un período de aislamiento a causa de la guerra mundial de 1939-1945 y la inmediata penuria posterior europea. En 1961 se perdieron las colonias de Goa, Damão y Diu; en ese mismo año empezaron los movimientos de independencia de Angola, y algo más tarde, los de Guinea y Mozambique. La industrialización del país y la reconstrucción de la Europa de posguerra provocaron, como en España, la gran emigración rural, acelerada en los años 60. El programa de «evolución en la continuidad» de Marcelo Cartano, sucesor de Salazar en 1968, no consiguió desarraigar la oposición al régimen, y en mayo de 1974, la llamada Revolución de los Claveles proclamó la Tercera República. Portugal terminó por conceder la independencia a las colonias, dando fin así al coste humano y económico que conllevaban las guerras sostenidas por evitarla.

Orlando Ribeiro ha padecido ese ambiente general de cambios. El paso por las calles lisboetas de soldados y cañones de los regimientos sublevados le acunó durante la Primera República y golpeó su sensibilidad en los años de colegio. En Lisboa veía pasar por la calle lavanderas cargando en la cabeza grandes cestas de ropa, y gallegos «moços de pau e corda» que «en tanto no hubo agua canalizada, en los barrios pobres donde me crié, iban a las fuentes públicas a rellenar sus cántaros pregonando en una encrucijada callejera ¡ahú!». Son, así escritas en castellano, sus palabras. Tenía por vecino un establecimiento que entre sus muros hermanaba, visibles al público, lechería y taberna, vacas y toneles, ordeño y escancia entre lonjas colgadas de bacalao. Y en 1918, con la población lacerada por la epidemia gripal, oyó por primera vez la palabra «bancarrota» en los comentarios que los mayores hacían sobre el empobrecimiento del país y la incompetencia de la Administración. El ambiente académico discurría consecuente con el socioeconómico, y durante la adolescencia y ya adulto, tuvo Orlando que soportar profesores 
sin vocación ni preparación. De tal maera que en la Universidad lisboeta, según ha escrito, «certas cadeiras de Geografia eram de uma nulidad desoladora, em História de um impudor total, pois o profesor ditava apontamentos traduzidos de livros que nos ocultava. Em Geografia o ensino era enteiramente teórico e verbal. Nunca fizemos uma excursão, nunca vimos um mapa de grande escala».Como eventual docente en la de Coimbra, padeció la pública censura por parte de un viejo profesor que recomendaba describir y no interpretar, como si esto, pensaba Orlando, hubiera que reservarlo a geógrafos extrajeros. Supo de colegas geólogos que no salían al campo y a quien abogaba por separar en la Universidad la enseñanza y la investigación. Y cuando ya empezó a ejercer en la Facultad lisboeta de Letras fue en un ambiente poco propicio: «Nume facultade velha e mal instalada, cada qual se escapulia depois de dar as aulas, uma vez que não existian gabinetes e lugares de convívio.»

«Remando contra a maré», según su propia expresión, supo despreciar la ganga y aprovechar la mena que le ofrecían los verdaderos maestros que encontró, a los que siempre tuvo presentes y para los que nunca ahorró palabras de gratitud. Que, como en los versos de Rilke, que recuerda según la traducción que de éste hizo Quintela, en la mente de Orlando «todos os verões, que nela calam, / agitam-se de novo nos mil ramos / e despertan de novo entre o Dia e o Sonho». A los cuatro años de edad quedó huérfano de madre y pasó a residir en el entorno de Viseu con su abuelo. De éste aprendió a observar la naturaleza y a gustar de la buena música y la lectura, en las que tan reconfortante terapia iba a encontrar después en sus momentos de depresión, enfermedad o vejez. A partir de los ocho años y de nuevo en Lisboa durante los períodos lectivos, completó la enseñanza primaria y cursó la secundaria, en la que, como más inclinado entonces hacia los estudios medievales, se matriculó en árabe para mejorarla cuando cursaba uno de los últimos años. Siendo alumno universitario de humanidades empezó a relacionarse y a conversar largamente con docentes de otras disciplinas. El primer gran viaje, efectuado en 1931, abarcó las provincias africanas portuguesas y Madeira, lo que amplió más sus conocimientos. En 1932, último año de la carrera, celebraron en la Facultad el centenario del fallecimiento de Goethe y correspondió a Orlando abrir las sesiones con una conferencia sobre la vida del poeta, lo que le obligó a profundizar en el personaje y a admirarlo más. Finalizada la licenciatura, descubrió que el organismo oficial encargado de la concesión de becas de estudio entendía que el 
aprendiz de investigador tenía que hacer una especie de prueba de entusiasmo para saber ganarse la vida de otra forma. Así, la adquisición de libros y la realización de viajes que necesitaba para su doctorado le obligaron a dedicar 32 horas por semana a las clases que impartía en un colegio.

Alcanzó el grado de doctor a los 25 años de edad con una tesis sobre la Sierra de Arrábida ${ }^{1}$. Marcello Caetano,director entonces del Instituto de Alta Cultura con el que había coincidido en aquella excursión científica y con el que mantuvo amistad durante los primeros años de carrera, le escogió para lector de portugués en la Sorbona. Una «das poucas pessoas a quem, fora dos mestres de meu ofício, fiquei a dever alguna coisa», reconocia Orlando. Pudo así proseguir éste sus estudios geográficos donde más deseaba hacerlo. Aprovechó la estancia parisina - el tiempo lectivo desde enero de 1937 a mayo de 1940 - para perfeccionar, en efecto, su formación. Y en el XV Congreso Internacional de Geografía, celebrado en Amsterdam en 1938, entró en contacto con ilustres colegas de otras latitudes. Al iniciarse la guerra mundial en 1939 quedó movilizado el profesor auxiliar que tenía de Martonne. Orlando le sustituyó hasta que, en el mes de mayo siguiente, quince días antes de que se produjera la ocupación alemana de París, regresó a Portugal, en cuya Universidad de Coimbra ejerció la docencia entre 1941 y 1943.

Cumplidos los 38 años fue nombrado catedrático en la de Lisboa, existente desde 1911. En esa situación inició la gran etapa de investigador estudiando la morfología del macizo antiguo portugués; tipos de poblamiento rural; vida pastoril de montaña con sus rasgos colectivistas; regímenes agrarios en Beira Baixa... Raro fue el año, decía, en que no trabajara al mismo tiempo en el Miño y el Algarve, en el Alentejo y en Trás-os-Montes, además de hacerlo en su «feudo» de la Beira Baixa, donde pasaba largas temporadas. Nombrado en 1945 colaborador de los Servicios Geológicos, dirigió levantamientos y colaboró en la confección de distintas hojas del Mapa Geológico a escala 1:50.000. En posesión ya de lo que consideraba razonable madurez, efectuó muchos otros viajes

${ }^{1}$ Ribeiro, O.: «A Arrabida. Esboço geográfico». Revista da Facultade de Letras. Lisboa. Universidad, 1937, IV, 1 y 2, pp. 51-137; id., id.: «Escursão à Arrabida» Finisterra. Revista Portuguesa de Geografia. Centro de Estudos Geográficos, Lisboa, 1968, III, 6, pp. 257-273 + 4 figs.; id., id.: A Arrabida, 2. $^{\text {e }}$ ed. Esboço Geográfico. Sesimbra. Ed. câmara Municipal de Sesimbra, 1986, 103 pp. 
con los que extendió más su horizonte cognoscitivo: a Grecia, el valle del Nilo, Italia, Marruecos y las áreas mediterráneas española y francesa. Recorrió el Sistema Central y las dos submesetas; los finisterres de Galicia, Asturias y Bretaña; los Pirineos y los viñedos de Aquitania; la llanura de Alsacia y el Jura; los Alpes occidentales y Suiza; la Inglaterra industrial del Black Country y la rural del País de Gales; las ciudades de Flandes y los polders holandeses; la Suecia central... También visitó con más atención las partes del mundo por las que se había extendido la influencia portuguesa: Madeira, Azores, islas de Cabo Verde y Santo Tomé, Guinea, Angola, Mozambique, India y gran parte de Brasil; y con más detalle, Madeira y Fogo, Goa, el Rocancavo brasileño y el sudoeste de Angola. Todo ello, con la intención de que, si no él mismo, sus discípulos, agregaran a los materiales recogidos los de Timor para elaborar un libro sobre Um Povo na Terra. Vendría a satisfacer su gusto por la obra de Camõns, presente en varias publicaciones de Orlando. La curiosidad siempre despierta por los fenómenos naturales lleváronle a estudiar las erupciones volcánicas de 1951 en Fogo y de 1957-1958 en Faial, ante alguna de las cuales se vio obligado a tomar decisiones en cuanto a la evacuación de la población, evitando a tiempo sufrimientos y muertes.

Dos hechos vinieron a estrechar más los lazos que le unían a la Geografía francesa, a la que debía su formación: la relación con la profesora Suzanne Daveau y la nueva estancia en Paris. Aquélla, autora de una tesis sobre las regiones fronterizas del Jura franco-suico, docente en las universidades de Dakar y Besançon y geomorfóloga en África occidental, se convirtió en su esposa, y en su colaboración encontró Orlando la interpretación de las fotos aéreas, que le estaban negadas por su falta de visión estereoscópica. Aclaraba el marido que esta «intimidade benéfica do trabalho acompanhase da indepêndencia crítica e, naturalmente, de respeto mûtuo das personalidades científicas de cada um». La nueva estancia parisina se produjo en el curso de 1967-1968, al ser invitado como profesor y asociado en el Instituto de Geografía de la Sorbona. La Universidad se había masificado y frente a quellos cinco profesores y un ayudante de 30 años antes, los mismos locales del Instituto habían pasado a albergar 17 de la primera categoría y 43 de la otra, y eran del todo insuficientes para la multitud de alumnos, situación generalizada que provocó la revuelta estudiantil de mayo del 68 , de la que Orlando fue testigo. 
Es cierto que las autoridades salazaristas le respetaron e incluso le solicitaron algún informe reservado sobre los problemas de Goa y la proliferación del chabolismo en el entorno de Lisboa. Y que recibió el elogio indirecto según el cual Salazar decía conocerle y apreciarle «por ser um homem que pensava pela própia cabeça e tinha muito boa cabeça para pensar». Pero tal aprecio, si es que en realidad existió, nunca estuvo acompañado de concesiones que pudieran mejorar las condiciones en que tenía Orlando que desarrollar su labor. Claro es que tampoco éste se mordía la lengua cuando creía necesario soltarla, que según recordaba en la lección que impartió con motivo de su jubilación, nunca se despreocupó de los aspectos morales que su actividad comportaba, por lo que alguna vez juzgó necesario tomar posición y lo hizo jugándose la carrera. Así, podía aseverar que «Considerado senão como un inimigo do Governo, pelo menos como uma pessoa incómoda, por dizer tudo o que me vinha à cabeça, se raras vezes fui perseguido, não tive junto dos poderes do estado a menor audiência». Tampoco la solicitó, porque sobre él pesaba el recuerdo de «a extinção da Facultade de Letras do Porto - primeira das muitas arbitrariedades e violências do governo de Salazar contra a inteligência da Nação». Y, lo que resulta paradójico, tras la llamada Revolución de los Claveles fue sometido Orlando a inquisitorial proceso «de saneamento» sin que consiguiera saber de qué se le acusaba $^{2}$.

\section{Los mentores}

Al igual que en España Dantín Cereceda —evocado por Orlando-, Martín Echeverría o Pau Vila durante los primeros decenios del siglo, también en Portugal hubo profesores anteriores empeñados en desbrozar con rudimentarios alpatanas: Barros Gomes, selvicultor que es-

${ }^{2}$ Su biografía personal y académica pueden verse con más detalle en Ribeiro, O.: «Cinquenta anos de vida científica e universitária». Revista da Facultade de Letras. Lisboa, 1986, $5 .^{\text {a }}$ serie, n. ${ }^{\circ} 6$, pp. 11-20, que es el discurso pronunciado el 16 de febrero de 1981 con motivo de su jubilación; id., id.: «Memórias de un geógrafo. Medio sículo de Ciencia e de Humanismo». Ensaios de Geografia Humana e Regional, I. Lisboa. Livraria Sá da Costa, 1970; id., id.: «Ciencia e Humanismo. Reflexiões sobre uma experiência». Broteria. Lisboa, 1983, n. $^{\circ} 117$, n. ${ }^{\circ} 4$, pp. 250-261; y n. ${ }^{\circ} 5$, pp. 396-415. Me he servido también de la versión de las Memórias de um geógrafo. Medio sículo de Ciência e de Humanismo, ordenadas por su esposa, la profesora Suzanne Daveau, a quien debo el favor de habérmelas facilitado. 
bozó los condicionantes naturales y humanos del tapiz vegetal portugués y los fundamentos de las tres grandes regiones del país; Silva Télles, primer profesor de Geografía en la Facultad a partir de 1904, sensible a la originalidad de los distintos paisajes; Amorim Girão, profesor en Coimbra desde 1918 y autor del atlas y una Geografía de Portugal, en la que veía la Península como encrucijada de caminos terrestres y marítimos donde, con frase que toma y traduce de nuestro Ángel Ganivet, «todos os povos e raças vieram distrair-se quando lhes pareceu oportuno, fazendo da história peninsular uma série interminável de invasões e de expulsões»...

Decía Orlando que «Uma carreira científica, vista a distância, está balizada afinal por pequenos factos decisivos». En efecto, hay que profundizar hasta fuentes más sólidas y complejas que ésas para descubrir los cimientos de su vocacion y su formación. Sus iniciales aficiones medievalistas se despertaron durante la adolescencia con las novelas históricas de Alejandro Herculano, crítico literario del teatro español y a quien le llegó la muerte, en 1877, en Vale de lobos, refugio similar y de igual nombre al que también iba a ser larga y última estancia de Ribeiro. Recordaba éste a Figanier, su profesor de geografía e historia en el instituto y causante de que el destino de Orlando al comercio - como había sido el del padre y en el que habría empezado de meritorio o mancebo- cambiara hacia la docencia. Sus lecturas eran entonces los Principios de Geografía Humana, de Vidal de la Blache, para él «constante apelo a duas fortes tendências do meu espíritu: la observação e a história»; y el Tratado de Geografía Física de De Martonne. Ya alumno universitario de Letras, frecuentaba la Facultad de Ciencias, en la que las lecciones se acompañaban de trabajos prácticos. Y, consciente de la necesidad de complementar su formación humanística con la naturalista, estudió en esos años a Humboldt, Darwin, Suess y Wegener. Leyó la Introdução ao studo da Medicina Experimental, de Claude Bernard, para Orlando lección de finura y exigencia de método en todo lo que concierne a la observación y la experiencia. Y, a la vez, cómo no, a Goethe, quien si le había emocionado cuando joven con sus obras de ficcion, también le admiró como teorizante del cráneo humano y cofundador de la anatomía comparada de los vertebrados. Entre los docentes universitarios destacaba a Leite de Vasconcelos, etnólogo, arqueólogo, filólogo, estudioso de las religiones primitivas de Portugal y organizador del Museo Etnográfico de Belén. De la primera estancia en la Sorbona re- 
cordaba especialmente los trabajos prácticos que dirigía De Martonne sobre Geomorfología con la lectura e interpretación de mapas; la riqueza de contenido de las de Siegfried, que sin ser un geógrafo, podían conducir al análisis regional; las de geología estratigráfica, de Jacob; las prácticas de campo de Baulig, profesor en la Universidad de Estrasburgo, y las conversaciones con Demangeon y con el mismo De Martonne, cuyo prestigio atraía a la Sorbona aprendices de geógrafo llegados de todas partes del mundo, aunque no de España, entonces en plena guerra civil. Ya docente universitario e investigador con experiencia fueron acompañantes suyos en las observaciones de campo su propio hijo, António Ribeiro, ahora catedrático de Geología en la universidad lisboeta; Mariano Feio, su primer ayudante; Lautensach; Solé Sabarís... Cuando el mismo Feio comentó que a Orlando no le importaba trabajar en el campo con cualquiera, él aclaraba: porque si sabe más que yo, aprendo; si sabe menos, gustosamente enseño; si sabe tanto, sometemos a discusión el problema que nos ocupa. Y, como auténtico maestro, tan deseoso de aprender como dispuesto a explicar, estuvo atento en todo momento a los nuevos descubrimientos o tendencias científicas sin desdeñar lo que, dentro de ellas, pudieran aportar sus propios discípulos. En tal sentido ha escrito: «Muito aprendi com os alunos e os que se inciaram comigo na investigação geográfica».

\section{Escritos y conceptos}

Dos de sus discípulos, primero, y Suzanne Daveau, después, se han tomado la molestia de relacionar los escritos del maestro ${ }^{3}$. Destacan dos aspectos generales: la gran capacidad de trabajo del autor , en relación con ésta, la vastedad de sus conocimientos. De esa capacidad dan fe las 375 referencias — de 1934 a 1995- de libros, artículos, comunicaciones a congresos, conferencias, recensiones, prólogos o simplemente reflexiones que integran el conjunto. Si en algunos casos se trata de reediciones, reimpresiones o traducciones a otras lenguas y de colaboraciones, las de los Opúsculos incluyen trabajos o evocaciones que

${ }^{3}$ Amaral, Ana, y Amaral, Ilídio do: Bibliografia Científica de Orlando Ribeiro. Lisboa, Centro de Estudios Geográficos, 1981, 85 pp, y Daveau, Suzanne: «Bibliografia Científica de Orlando Ribeiro (2. ${ }^{a}$ parte, 1981-1995)", Finisterra, 1996, XXXI, n. ${ }^{\circ} 61$, pp. 87-97. 
permanecían inéditos. Y la amplitud temática se refleja en la variedad. Junto a los dedicados a sus maestros, directos o no, humanistas o naturalistas y en especial a geógrafos -Pierre Birot, Lautensach, Francisco Hernández Pacheco, Manuel de Terán...-, aparecen abundantes estudios geomorfológicos o geológicos: «Paysages à Inselberg»; el relieve de Angola entre el Zaire y el Loge; las hojas de Nisa, Castelo Branco, Abrantes y Gavião de la Carta Geológica a escala 1:50.000; A Ilha do Fogo e as Suas Erupções; etc. También algunos de clima y vegetación, como el del ritmo climático y el paisaje; el algarrobo o Ceratonia sili$q u a$, el trigo, el olivo, el mijo y el maíz, en relación con la expansión y el cultivo; otros de población y numerosos de temas rurales; los de las ciudades de Lisboa, Oporto, Evora, São Paulo, Viseu y el de su «Rua Direita»; los regionales, tan de su agrado, tales el de las islas atlánticas portuguesas, la tipologíaa del paisaje rural americano, la organización del territorio en África occidental y su agricultura, Brasil y la regionalización de su espacio, Goa, Guinea, Madeira, etc. Es decir, unos referidos al Portugal europeo, otros a los espacios de colonización lusitana.

Con una observación más detallada cabe agrupar los estudios de Orlando en cuatro características o tipos: los de minuciosa investigacion analítica, los sintéticos que se apoyan en ella, los conceptuales que los encarrilan y los didácticos que los transmiten. En el primer grupo se hallan casi todos los arriba mencionados: la simple indicación de sus títulos escapa a la simplicidad de este bosquejo. Gracias a ellos y a la capacidad de síntesis del autor pudo éste escribir su Portugal, o Mediterrâneo e o Atlântico, con tanto éxito que cuenta ya siete ediciones, y el tomo quinto de la Geografía de España y Portugal que dirigió Terán y publicó Montaner y Simón en 1955. Finalmente, en tal sentido, los cuatro volúmenes - dedicados sucesivamente a la posición geográfica y el territorio, al ritmo climático y el paisaje, al pueblo portugués, y a la vida económica y social- de la gran Geografía de Portugal, en cuatro volúmenes, cuya organización unitaria, actualización e incorporación de algunos capítulos debidos a la pluma compartida de Ribeiro y Lautensach se deben a Suzanne Daveau. Fruto de los viajes del maestro por los países circundantes del mare nostrum es también su tratado sobre $O$ Mediterrâneo, Ambiente e Tradição, y del que realizó por Europa, la Introdução a o Estudo da Geografia Regional. Son los más destacados dentro de los que, a pesar de la amplitud, cabe englobar en el apartado de sus trabajos de síntesis. 
Los conceptos que Orlando tenía sobre la Geografía aparecen, al menos de manera implícita, tanto en aquellos trabajos analíticos como en los sintéticos. Más explícitamente, en distintos artículos que fueron apareciendo a partir de 1941: «Orientações modernas da Geografia», «Meditações Geográficas», «Acerca de alguns conceitos fundamentais da investigação científica», «Rigor e refleção na ciência moderna», «O oficio de geógrafo» - después reeditado en revista francesa—, «Nótula sobre a "inutilidade" da Ciência», «Geografia y reflexão filosófica», el titulado "Ciência e humanismo. Reflexões sobre uma experiência»... Consideró desde el principio que la Geografía es una rama más de la Ciencia y que, en consecuencia, está delimitada por otras cuyos conocientos generales no debe ignorar el geógrafo. Que es bueno que cada uno, como señalaba Goethe, sepa distinguir lo que en la naturaleza es real y trascendente. Porque el geógrafo, al igual que el naturalista, debe saber que el mundo físico y biológico está en contínua transformación, pero que, comparado con la brevedad de una vida, es algo que permanece. Que «A geografia é, ao tempo, uma ciência de base e de convergência, um ponto de partida e um lugar de encontro: como uma encruzijada, portanto, onde se chega e donde se sai por varios caminhos»; con dos supuestos fundamentales: el amor a la naturaleza y el gusto por la Historia. Se alegraba así de que, siendo radicalmente humanista, hubiera adquirido a la vez una formación de naturalista, indispensable al geógrafo; que, a su esencial humanismo, hubiera unido la entrañable vocación de amante y observador del medio físico. Y, a la vez, entendía que todo lo que se refiere al hombre es una manera de ver y sentir lo que ha pervivido de la Historia entre nosotros, que el presente proviene del pasado. Porque para él, según destaca Baulig en el prólogo a los Opúscu$l o s$, nada del paisaje ha de sernos indiferente. Esa simbiosis entre elementos históricos y naturales subyace en toda su obra porque inseparable estaba en su espíritu. Cabe recordar como ejemplo que cuando fue a observar las erupciones de las islas atlánticas no dejó de anotar también las formas de vida - las peligrosas formas de vida- de quienes vivían - de quienes malvivían - al pie de los volcanes.

Eso no quiere decir que fuera ajeno a los cambios e innovaciones que fueron surgiendo en el quehacer geográfico. Comprendía que los graves problemas del mundo actual hayan provocado la atención de muchos colegas. Y que todo geógrafo bien preparado puede ser útil, mejor que otro, en tantos trabajos de grupo que se dejan en manos de ingenieros y ar- 
quitectos, cuando no en las de abogados, sociólogos y economistas de gabinete. Aunque viendo la Geografía aplicada con alguna prevención, no dejó de tratar sobre la planificación regional y la organización del espacio ni de tomar parte en la comisión encargada de aprobar el plan rector de la región de Lisboa. En el segundo período de estancia en la Sorbona detectó el desinterés por la Geografía regional que los colegas franceses tanto habían prestigiado; la ruptura entre los estudios de la naturaleza y los humanos, frente a la concepción vidaliana de «não parcelar o que a natureza junta, a compreender a correspondência e a correlação dos factos, quer no ambiente terrestre que os envolve todos, quer nos ambientes regionaiss em que se localizam». Veía que el sensacionalismo invade y nubla el claro cielo de la Ciencia y que aparece así una llamada geografía activa, como si el trabajo científico desinteresado no fuera siempre resultado de la voluntad y la acción. Parece con ello, decía, que se quisiera significar que el geógrafo puede o debe actuar e intervenir en decisiones que, en definitiva, corresponden al poder político concebir y ejecutar. Y encontraba ese nuevo quehacer geográfico apenas atento al presente y volcado sobre las incerditumbres del futuro sin disponer de método propio de previsión y subestimando o repudiando la Historia. Como si el geomorfólogo tratase de interpretar el relieve e imaginar su futura evolución haciendo tabla rasa de los movimientos tectónicos, de las oscilaciones del nivel de base y de las vicisitudes del pasado. Pero tampoco dejó por ello de apreciar la renovacion de la biogeografía, antes apenas cultivada por algunos botánicos de orientación geográfca o por raros geógrafos con preparación especial. $\mathrm{O}$ el papel concedido cada vez más al conjunto de los procesos morfogenéticos, de los paleoclimas, los suelos y la vegetación. A esta ampliación del campo de nuestra disciplina responde su «Tradición e innovación en el sistema de la Geografía».

Pero Orlando era, además de investigador, un vocacional docente y, como tal, siempre estuvo preocupado por educar - esto es, conducir- a sus discípulos, despertar en ellos el interés por la Geografía como una rama más de la Ciencia. Entendía que docencia y mediocridad forman un matrimonio estéril. Y que, a escala universitaria, la docencia y la investigación son inseparables. Que «Uma escola científica não é repetição dos mesmos modelos, mas a arte de façer com que cada um revele a personalidades mais adequada ao seu espíritu, à sua formação e à propia alegria do trabalho pessoal». En esta cuarta característica o 
de la orientación cabe encuadrar el Inquérito de Geografía Regional y su Inquérito do habitat rural, ambos publicados en 1938 y, después, en segunda edición; y otros muchos que siguieron a ésos. Le preocupaban los problemas de la Universidad, y esa preocupación le llevó a redactar «A universidade e o espíritu científico», la «Inquietação e esperança na Universidade», «O problema fundamental da Universidade», la «Universidade selectiva», «Universidades sem professores» y varios más de similar guisa. $\mathrm{Y}$, los dedicados a los profesores de institutos, aquéllos y éstos en periódicos o en revistas didácticas. Quería llevar al ánimo de esos profesores que la Geografía, como toda ciencia, es útil por su llamada constante a la reflexión, muestra la variedad terrestre y humana e inclina el espíritu a la comprensión, la equidad y la tolerancia, sentimientos de los que los pueblos más carecen para establecer entre ellos una convivencia posible. Podemos decir con expresión unamuniana que a Orlando le dolía Portugal. Así, dentro de esa inquietud por educar cabe encuadrar los escritos en los que denunciaba también la mala gestión llevada en las colonias: «Descolonização, ensino e ciência», «Destinos do Ultramar», «Dificultade da Independência», «A colonização de Angola e o seu fracasso»... Y la destrucción, como en España, del patrimonio natural, dolor explicitado en su «Portugal arde por toda parte».

\section{Las creaciones}

En 1943 creó Orlando el centro de Estudios Geográficos en la Facultad lisboeta de Letras y en él fue director durante casi tres decenios. Difícil resulta resumir la labor que ha realizado y realiza este polo irradiador y de servicio o apoyo a la enseñanza y la investigacion. Basta mencionar lo que se manifiesta en el breve folleto conmemorativo del medio siglo de vida. Señala seis áreas de investigación, líneas de actuación o proyectos en las que el Centro está comprometido: estudios para el planeamiento regional y urbano, geografía humana y regional, Geografía física, ambiente y ordenación biofísicos y Geografía regional e histórica. También, entre otros, los cursos del master, uno sobre Geografía física y medio ambiente, otros de Geografía humana y planificación regional y local, aquél con 15 asignaturas y el segundo con 11 . Y los gráficos, correspondientes a otros tantos trabajos, que lo ilustran: el del

$$
-386-
$$


reparto de frutos secos y sus derivados, perteneciente al denominado $O$ Algarve Oriental, del que es autora la profesora Carminda Cavaco; las unidades de ocupación del suelo, parte del titulado $A$ Ilha do Corvo, de Carlos Alberto Medeiros; los flujos aéreos entre las principales ciudades de Europa Occidental, con número de enlaces entre tres y diez vuelos diarios, que se incluye en el estudio sobre Rede Urbana Europeia, de un equipo que dirige Jorge Gaspar; el mapa de isocronas a partir de Evora, parte del de A Area de influência de Evora, del mismo profesor Gaspar; el esbozo morfológico de Ilha Graciosa, realizado por António Brum Ferreira, todos ellos en blanco y negro. Más los reproducidos a todo color de los Contrastes Térmicos, Nevoeiro e Nebulosidade de Portugal, del equipo dirigido por S. Daveau; el plano de Edifícios do Bairro Alto segundo o número de pisos, de J. Gaspar y colaboradores, y Desvios de temperatura [en Arrábida] em relação á estaçao de Setubal, de J. M. Alcoforado. El Centro contaba en 1993 con 59 investigadores, 19 doctores, 21 profesores - casi todos también en la Universidad-, y 19 licenciados. Y la biblioteca, con 30 millares de títulos, riquísima cartoteca y medios mecánicos de informatización ${ }^{4}$.

A los seis años de la creación del Centro tuvo Orlando que organizar el XVI Congreso Internacional de Geografía en Lisboa «mendigando subsídios, fazendo despensas a crédito ou adiantando, do meu magro vencimento de professor, o dinheiro indispensável para o gastos correntes». Casi sin otra ayuda que la de Fernandes Martins y Mariano Feio -todavía entonces únicos geógrafos activos-y la de algunos colegas de otras especialidades, como los geólogos Zbyszewski y Teixeira, la historiadores Vigínia Rau y el etnólogo Jorge Dias. Aún sacó tiempo para aportar cuatro comunicaciones y dirigir dos de las excursiones, las del Portugal central y la isla de Madeira. Y a fe que el Congreso se desarrolló con pleno éxito, que la UGI reconoció otorgando a Ribeiro una de sus vicepresidencias. Se incribieron 800 investigadores pertenecientes a 37 distintos países y, entre las comunicaciones presentadas, una docena estaban firmadas por españoles. La publicación de todas comprende cuatro volumenes - uno de ellos doble- y fue viendo la luz a partir de $1950^{5}$.

${ }^{4} 50$ Anos de Investigação Geográfica. Lisboa. Centro de Estudos Geográficos. S.A., $21 \mathrm{pp}$.

${ }_{5}^{5}$ Union Geographique Internationale: Comptes Rendus du Congrès International de Géographie. Lisbonne, 1949. Lisboa, 1950-1952, 4 volúmenes. 
Otra creación de Orlando a destacar es la de la revista geográfica $\mathrm{Fi}$ nisterra, editada por el mismo Centro de estudios Geográficos, que desde 1966, en que apareció el primer número, no ha dejado de publicarse hasta ahora. En la presentación de aquél, titulada «Orientação» señalaba los objetivos; «fazer de Finisterra, não un lugar isolado da Ciência mas una janela aberta o mundo - que, sendo afinal o campo de trabalho de todos nós, é também o sítio do nosso encontro». $\mathrm{O}$, según expresaba en el artículo incluido en otra revista de cultura e información en número coetáneo a aquél, vehículo de reflexiones e investigaciones a partir «doutro ponto de vista no panorama geral da Ciência dos nossos dias».

No todas sus empresas se coronaron con similar éxito. Es lo que ocurrió con su proyecto de vitalizar las enseñanzas humanísticas ante la reforma universitaria de 1957. Porque, decía, la triste verdad es que la Universidad, y en especial las Ciencias Humanas, tanto entre los poderes públicos como entre el público en general, no tiene prestigio, voz activa ni fuerza para imponerse. Sin embargo, el Centro de Estudios Geográficos ha continuado desarrollándose bajo su dirección y la de sus sucesores hasta alcanzar las cotas que pueden deducirse con lo ya expuesto. Y los Coloquios Ibéricos que iniciamos tímidamente en Salamanca en 1979 siguen practicándose alternativamente en ambos países peninsulares. Así, la figura de Orlando, su calidad docente y su incansable labor científica son reconocidas por todos. De tal reconocimiento dan fe aquella vicepresidencia de la UGI, los cinco doctorados honoris causa recibidos - de las universidades de Río, Burdeos, Coimbra, Complutense y Sorbona - y la francesa legión de honor que le fue concedida. Pero, sobre todo, la pléyade de discípulos a los que entusiasmó, encaminó hacia la Geografía y mantienen el prestigio de la escuela portuguesa para la que Orlando empezó a abrir los surcos rectores hace algo más de medio siglo. 